\title{
Practical Monitoring of Resource Utilization for HPC Applications
}

\author{
Antonio Gómez-Iglesias, Carlos Rosales, Todd Evans \\ Texas Advanced Computing Center \\ The University of Texas at Austin \\ Austin, TX \\ \{agomez, carlos, rtevans\}@tacc.utexas.edu
}

\begin{abstract}
HPC centers run a diverse set of applications from a variety of scientific domains. Every application has different resource requirements, but it is difficult for domain experts to find out what these requirements are and how they impact performance. In particular, the utilization of shared resources such as parallel file systems may influence application performance in significant ways that are not always obvious to the user. We present a tool designed to provide the information that is most critical for running an application efficiently on HPC systems. The information provided forms a complete view of the application's interaction with the system resources, which is typically missing from other profiling and analysis tools. The tool is designed to be scalable and have minimal impact on application performance, and includes support for different accelerators.
\end{abstract}

\section{CCS Concepts}

-Human-centered computing $\rightarrow$ Open source software; -Software and its engineering $\rightarrow$ Monitors; Massively parallel systems;

\section{Keywords}

HPC; resources; monitoring

\section{INTRODUCTION}

HPC systems are used by a variety of domain experts, students, and researchers that are not computational experts. However, neither the technology nor the tools they use are designed with them in mind. This creates a disconnect between users and resources that limits the productivity of these systems. Tools that would be of help to users in improving their execution times are often accessible only to system administrators and support staff.

Examples of this can be found in the area of monitoring and profiling tools. Profilers are typically designed to provide a high level of detail about a given application. They

Permission to make digital or hard copies of all or part of this work for personal or classroom use is granted without fee provided that copies are not made or distributed for profit or commercial advantage and that copies bear this notice and the full citation on the first page. Copyrights for components of this work owned by others than ACM must be honored. Abstracting with credit is permitted. To copy otherwise, or republish, to post on servers or to redistribute to lists, requires prior specific permission and/or a fee. Request permissions from permissions@acm.org.

XSEDE16, July 17-21, 2016, ,

(C) 2016 ACM. ISBN 978-1-4503-4755-6/16/07 . \$ $\$ 15.00$

DOI: http://dx.doi.org/10.1145/2949550.2949643 can collect a wide variety of data and correlate this data to specific sections of the code. While this is useful for many users, it is also the case that many other find profilers overwhelming and difficult to understand.

Even with the complexity of these tools, it is often the case that the most common questions that users have still remain unanswered. This is something that HPC consultants can see in their interactions with the users of HPC environments. A typical question is how much memory a particular application consumes on each one of the nodes used during its execution. While this seems to be a trivial problem for advanced users of HPC resources, it is often a constraint for other users that are not able to identify why their simulations fail.

This paper describes a new tool that helps users understand how their applications interact with the different hardware resources of HPC systems. This tool monitors those resources for each one of the compute nodes utilized by the application and generates a summary that is accessible to users with all levels of HPC experience.

The rest of this paper is organized as follows: Section 2 describes the tool that we present, while Section 3 details the implementation and the different configurations that the tool provides to users. Then, Section 4 presents different use cases and how the data collected by our tool can be used to improve the utilization of HPC resources. Section 5 presents the related work and, finally, Section 6 concludes the paper and presents some ideas that will be considered in the future to extend the current functionality of the tool.

\section{MONITORING TOOL}

REMORA (REsource MOnitoring for Remote Applications) $[1,2]$ is a tool designed to provide HPC users with practical information about the resources used by their applications. Running an application with this tool results in a straightforward summary that characterizes the resources most important to effectively running that application on an HPC system. This summary focuses on accessible information such as CPU and memory utilization, I/O (Input/Output) load, and NUMA (Non-uniform memory access) statistics. The tool does not depend on any external library or application, and it presents the collected data to the end user as it is gathered.

Unlike system-wide monitoring tools, our approach focuses on single job analysis that allows for fine-grained data collection without affecting overall system performance. On the other hand, unlike traditional application profilers, this tool provides data that correlates directly with the available 
hardware resources in a way that is simple to understand. The comprehensive nature of the data collected and the direct correlation to system properties form a powerful combination that allows users and system administrators alike to evaluate quickly the possible inefficiencies in the way an application is run.

When compared to resource monitoring and application profiling tools, the tool that we present in this paper is unique in several aspects: 1) ease of installation and use; 2) comprehensive resource monitoring; 3) simple user extension capabilities; and 4) real time access to monitoring data.

Many profilers are designed in a manner that requires users to have a substantial knowledge of computer fundamentals in order to effectively interpret the profiling results. Interpreting hardware counter data provided by these tools can be a daunting prospect even for experienced researchers. Moreover, the information that they collect aims at providing specific insights about how the application has been developed, not about how the application interacts with the different computational resources used during the calculations. Many users of current HPC systems are domain experts that do not have that type of knowledge or the time to build it, and must prioritize their efforts on producing the results most relevant to the scientific problems they are funded to study. However, these same users would naturally like to get the most out of the computational resources in order to increase their scientific productivity. Our tool addresses these needs by providing readily accessible and practical information. REMORA presents that information in such a way that users can quickly get an overview of the main characteristics of their applications as well as some of the resource limits they are encountering. Examples of these limits include local memory limitations, limitations on metadata requests to the shared file system servers, and bandwidth limitations between nodes.

Our tool can also be configured by system administrators to prevent potentially problematic jobs from overextending available computational resources. Consider the metadata example mentioned above, where it is well-known that large numbers of file operations can result in file system server hangs and occasionally crashes that create system-wide effects. While most community HPC applications will not cause these issues, applications that are still in development or have never been run on HPC systems often were not designed to account for such limitations. With the appropriate configuration REMORA can be provided to users to safely test the resource demands of their applications and stop the execution if a given set of conditions, i.e. metadata request rates exceed a configurable threshold, before system-wide effects manifest.

While traditional profilers introduce a significant overhead in the execution of the application of interest, the tool that we present in this paper has a very low overhead, with minimal impact on the overall performance of the application and requiring little in the way of system resources. Since REMORA is intended to work with serial and parallel applications, this low-impact approach is a critical element to ensure the scalability of the tool. In our tests, REMORA can be used with large-scale applications with minimal impact on their performance.

REMORA is currently installed on the most heavily utilized clusters available at the Texas Advanced Computing
Center. It is used by both end users and administrators to find problems with jobs that the users submit to those clusters as well as to get additional insights that can help improve the performance of the scientific applications.

\section{DESIGN OF THE TOOL}

The monitoring component of REMORA has been implemented entirely in Bash. This ensures its portability and ease of installation. The tool can be installed without root privileges and can reside in user space. Thus, users of different HPC resources can simply download and use the tool without requiring the intervention of system administrators. The analysis component requires a basic Python installation but can be performed offline. As detailed in Section 3.5, the tool follows a modular design to ensure its extensibility and portability to other platforms that are not currently supported. The modules REMORA is composed of are sourced during the initialization of the tool, so that a set of required functions are loaded and defined.

\subsection{Configuration}

The tool provides a limited set of options that allow users and administrators to configure the default behavior.

The tool supports two modes of operation. In basic mode, it only collects information about the CPU and memory utilization of the application. In full mode, the tool will collect all the data described in Section 3.2 as well as any extra information that is gathered by new modules implemented by the administrators or the users.

The elapsed time between two consecutive data collections can also be customized. For long-running applications, it is recommended to use a value of 10 seconds or higher to reduce the overhead of the tool. For these long-running applications, it is not normally necessary to have very fine resolution, as collecting this type of data more frequently rarely provides additional understanding of the application of interest. In addition, the size of frequently collected data may be quite large for long-running applications.

\subsection{Statistics Collected}

We currently collect the following information:

- Timing of the application: this time includes the overhead introduced by the tool. We do not collect linelevel data or data for specific functions or modules.

- CPU utilization: the tool collects the CPU utilization of all the cores on each node used by the application. This data can be used to find load imbalances.

- Memory utilization: this data is often what users are most interested in. REMORA gathers the utilization of virtual and resident memory, as well as RAM file systems. It also displays the peak memory used, which is what can trigger the invocation of the OS's out of memory killer.

- NUMA information: this is critical information for OpenMP applications. Often, developers of these applications do not take into consideration the performance implications of NUMA architectures. The data collected will show NUMA misses as well as imbalances in the memory allocation within each node. 
- I/O information: poorly performing file access patterns represent one of the most difficult and overlooked challenges for HPC applications, but they are often difficult to identify. REMORA currently provides Lustre statistics that help to identify high loads on the I/O servers that may indicate excessive file system activity.

- Network information: the tool records the network topology as well as the InfiniBand traffic generated by the application. The network topology can be used to identify slow elements in the network which may be of significant value to system administrators.

\subsection{Monitoring of Serial and Parallel Appli- cations}

Once the environment has been properly set, users only need to prepend the command they would normally execute with the remora command. For example, remora mpirun parallel_application and remora serial_application. This will launch the main REMORA script. This script will initialize the tool and perform a set of checks to ensure that the operation that the user wants to perform is correct. After that, it will read the REMORA configuration and initialize the modules specified in that configuration.

Once the modules have been initialized, the tool will detect how many nodes have been allocated for the current job and will start a background process on each of those nodes that will run the different modules responsible for data collection.

Immediately after all the background tasks have started, REMORA calls the main application (i.e. mpirun parallel_application or serial_application). Once the application has finished, we finish all background processes and perform any post-processing that might be required on the compiled data.

As described earlier there is no difference in the way the tool is invoked for parallel and serial processes. Internally the only difference will be that data is collected for each node separately and then aggregated as necessary to provide a sensible summary of the resource utilization, but this process is transparent to the user. Notice also that there is no need for the user code or workflow to be recompiled or changed in any way.

The way we currently track user processes means that certain data such as memory utilization is reported per node rather than per process ID. Practical experience with the tool indicates this is a sweet spot for a good compromise between the time spent collecting and analyzing data and the usability of the data itself. However, extending the tool to provide more fine-grained data in terms of individual processes run is perfectly possible if future feedback indicates this is a desirable feature.

\subsection{Hybrid Platforms}

Nowadays it is common to find HPC resources that include some type of accelerator. Because of the popularity of these resources and the number of codes that take advantage of those devices, REMORA includes modules for collecting statistics in environments that use GPUs and/or Intel Xeon Phi coprocessors. The design of the tool makes it easy to expand it to support new architectures as well as tools that allow for the collection of new types of data.

GPUs.
The tool uses nvidia-smi to collect the memory that is currently used as well as the available memory. Capturing power utilization is possible via the same interface but has not yet been implemented in REMORA. Since GPUs do not allow for remote access, all interactions have to be performed from the host CPU and thus the performance of the data collection is limited by both the vendor provided tools and the CPU/GPU interaction. Overhead of data collection from GPUs is the highest of all operational modes in REMORA, and it is recommended only for development and debugging purposes.

\section{Intel Xeon Phi.}

REMORA has been tested on Stampede ${ }^{1}$ at the Texas Advanced Computing Center. This cluster was the largest Intel Xeon Phi deployment when it became available in 2013, with at least one Intel Xeon Phi installed on each of the 6400 nodes. REMORA collects the memory usage of each Intel Xeon Phi used by the application and presents very little overhead when the background collection task is pinned to the very last core in the coprocessor - the same that Intel uses to run the COI (Intel Coprocessor Offload Infrastructure) daemon.

\subsection{Modular Design}

REMORA presents a modular design that makes it easy for users and system administrators to modify and extend the functionality provided by the tool. As previously described in Sec. 3.2, REMORA already collects a number of statistics. However, these statistics might be extended or replaced by other data. For example, currently the tool supports the Lustre file system, but there are HPC systems that use other types of file systems. Data collection for those file systems can be incorporated into REMORA in a straightforward manner due to its modular design.

This design allows the automatic discovery of new functionality by means of a configuration file that is read during the initialization of the tool. This configuration file contains a list of module names to activate. Typically, each of these modules collects a different type of statistic. The tool will read each line and will load a script file with the same name specified in the configuration file. The script file needs to implement at least four functions: initialization, data collection, post-processing, and finalization.

As explained in the next section, the functionality already implemented in REMORA takes into account performance and scalability issues so that the overhead introduced by the tool in the overall execution is low. While all the current functionality is implemented in shell scripts, developers can easily extend the functionality of the tool by implementing new modules in any programming language.

\subsection{Usability}

REMORA presents an easy-to-use commandline interface. Users only need to use one command for data collection and another command in case they need a postprocessing step. This postprocess generates a set of plots with the data previously collected so that the results can be easily visualized.

For data collection, the user simply needs to prepend the regular command that would be used to run a given appli-

\footnotetext{
$\overline{{ }^{1} \text { https://www.tacc.utexas.edu/stampede/ }}$
} 
cation with the command remora. This remora script will create $s s h$ connections to each one of the nodes involved in the computation and launch a background process on them. This background process will monitor the various resources supported by REMORA. The data is stored in a folder in text format so that users can easily access all the information.

Once the main application has finished and the data has been collected, a Python script can be called. This script will look for the folder with the different statistics. It will create a plot for each of the elements previously monitored i.e. memory, or CPU utilization - on each one of the nodes used during the computation.

\subsection{Performance and Scalability}

The functionality implemented in REMORA introduces a small overhead in the execution of the target application. The data that the tool collects is accessible through system logs and performance counters that are available to all the users of the system. The current version does not gather data that needs special privileges to be collected, although this could be easily changed.

When reading the different counters that are currently used, we try to reduce the number of operations as well as data handling that we perform to reduce the requirements of the tool. Most of the data is extracted from the /proc file system. One of the most complicated data collection cases is the case of the Lustre counters (see Alg. 1). These counters are located at /proc/fs/lustre/\{mdc,llite\}. When reading those counters, the names of the file systems might not match what the users see. When developing a tool that automatically captures all this information and tries to present the data in an easy to understand manner, it is critical that the users see the results exactly how they expect them. In order to correlate the internal name of the file systems with the name where the file system is mounted, we use the output of the df command. Since some processing is required to extract the relation between file system and mount, we only perform this operation during the initialization of that module. Finally, in the case of Lustre counters, each node used during the execution of the application will create a file for each file system with the load created by that node on the Lustre servers. The finalize_module method will aggregate the data from all the nodes into a single file. This file will contain the I/O load generated by all the processes involved in the application as well as the volume of read and write operations. Although REMORA does not currently collect statistics for other file systems, the procedure to collect the data in other systems should be similar to the one described.

As shown in Alg. 1, there are at least three functions that are called for each module. In terms of performance and overhead, it is critical that the collect_data_function has minimal computational requirements since it is called during the application's execution. The other two functions are called only once, and they contribute little to overhead. For example, although the FINALIZE_MODULE_LUSTRE function in Alg. 1 processes a large number of files it is called only once, after the application has run. These functions can have a relatively large impact in the overall execution time for short applications but their total execution time should never exceed a few seconds.

A careful study of the runtime overhead introduced by the

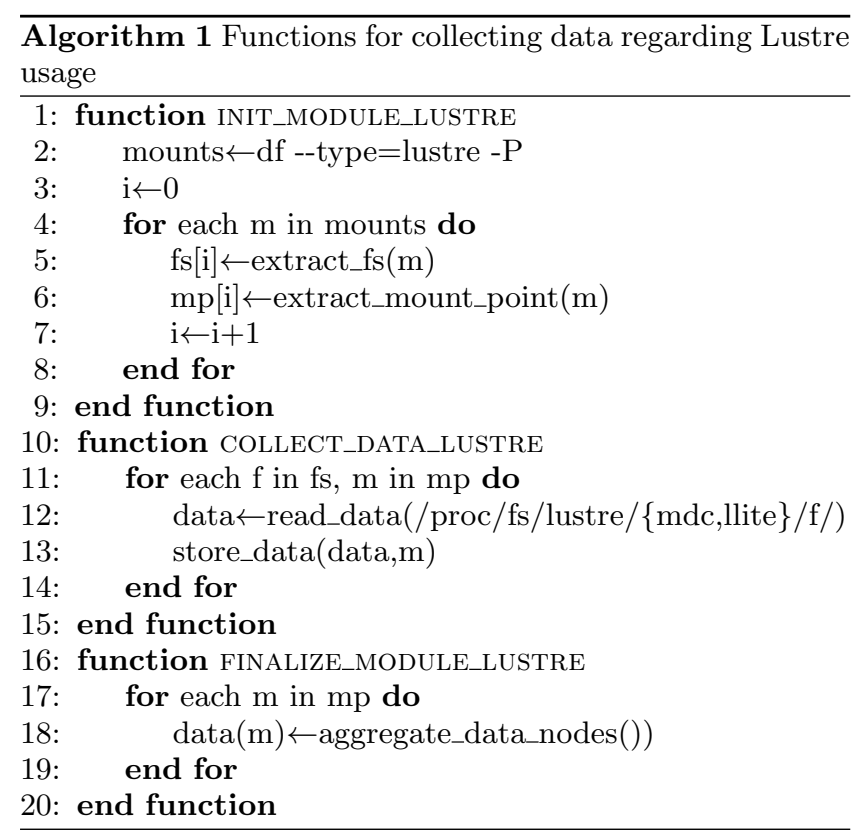

functionality currently implemented in REMORA has been performed for different collection settings. The code chosen to perform the scalability and overhead tests is part of MP-LABS [3], an Open Source implementation of the Multiphase Lattice Boltzmann algorithm. The code has minimal I/O and exercises both CPU and memory subsystem, representing a worst case scenario for overhead issues when running simultaneously with REMORA. A constant workload was maintained per process in all tests, and ten repetitions were performed for each test before calculating the average runtimes. The overhead is reported in $\%$ from the formula:

$$
\% \text { Overhead }=\frac{(R E M O R A-B A S E L I N E) \times 100}{B A S E L I N E}
$$

Fig. 1 shows the overhead introduced by running the tool in the two currently available modes (BASIC and FULL) and different values for REMORA_PERIOD (1, 10 and 30 seconds). It can be seen that for runs on a single node (16 processes) the overhead introduced by REMORA is below $1 \%$ when using sampling intervals over 10 seconds, and reaches $4 \%$ for sampling intervals of 1 second. The exact numbers are provided in Table 1. Even when scaling to over a thousand processes the overhead remains well below $10 \%$ for sampling periods of one second, and below $7 \%$ for sampling periods of 10 seconds or more.

\begin{tabular}{l|c|c}
\hline Mode & Period (s) & Overhead (\%) \\
\hline BASIC & 1 & 3.8 \\
BASIC & 10 & 0.7 \\
BASIC & 30 & 0.3 \\
FULL & 1 & 4.1 \\
FULL & 10 & 1.0 \\
FULL & 30 & 0.5 \\
\hline
\end{tabular}

Table 1: Overhead for single node execution of REMORA

In some cases the code appears to run faster when we col- 


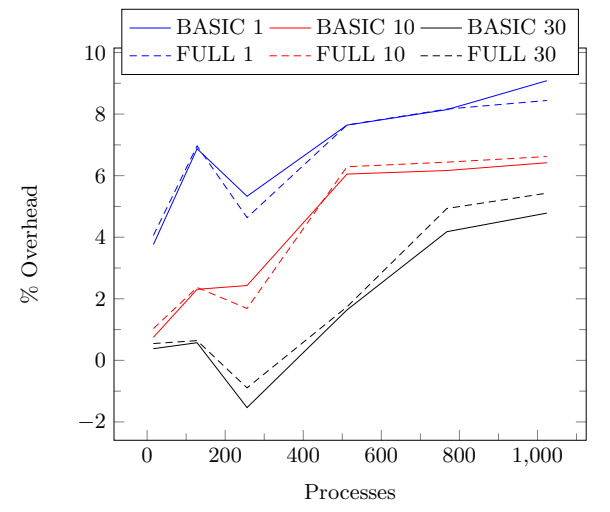

Figure 1: Overhead for different sampling periods and number of processes

lect data than when running in baseline mode. This is most likely due to caching issues, and also indicates that the overhead of the tool is comparable to the system variability. It is also important to note that adding the collection of Lustre file system data and network topology does not significantly increase the overhead, making this the recommended mode of operation.

\subsubsection{Intel Xeon Phi}

As previously mentioned, we collect data regarding memory usage on the Intel Xeon Phi co-processor for those applications that use it. Intel Xeon Phi cores are very limited compared with the most popular cores that can be found in most of the nodes of current machines. This is critical when designing an approach with low overhead and good scalability. In order to limit the impact on the performance when using Intel Xeon Phi, we bound the task that collects information on the co-processor to the core 61 . This core is normally not used by the application since it is reserved to the operating system and to perform data transfers between the host and the Intel Xeon Phi.

\section{USE CASES}

In this Section we present a number of typical use cases that can be found on HPC systems and are representative of situations where REMORA helps users to improve the performance of their codes as well as ensure a better use of the computational resources. Users can extract additional information from the data that we collect, combining the statistics we collect with knowledge they might have about the application. In the future we plan to provide analysis agents that will comb the collected data for known or potential issues and highlight those to the user.

Fig. 2 shows the I/O load in terms of number of I/O operations per second performed by a serial Python code running on a Lustre file system. This profile represents a very typical scenario, where the program performs some computation and writes the results to a file on each step of the algorithm. Many times, developers are able to change the code so that the results are only written after several steps or at the end of the computation. This not only reduces the load on the file system, but also enhances the performance of the application. Since REMORA collects the information regarding the system usage, the language used to implement the application does not pose a limitation for the tool.

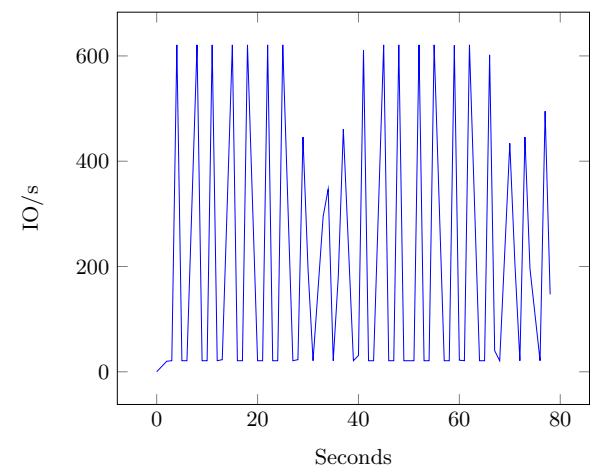

Figure 2: MDS load created by a serial application

Fig. 3 shows a case where two of the OSTs (Object Storage Target) available (the figure only depicts 5 OSTs instead of all of the OSTs in the system) are used during the execution of a job. In this case the number of stripes used was 2 . For large executions with heavy I/O, it is recommended to increase the number of stripes to better distribute the load. REMORA is able to capture this level of detail and the users can quickly identify possible problems regarding striping.

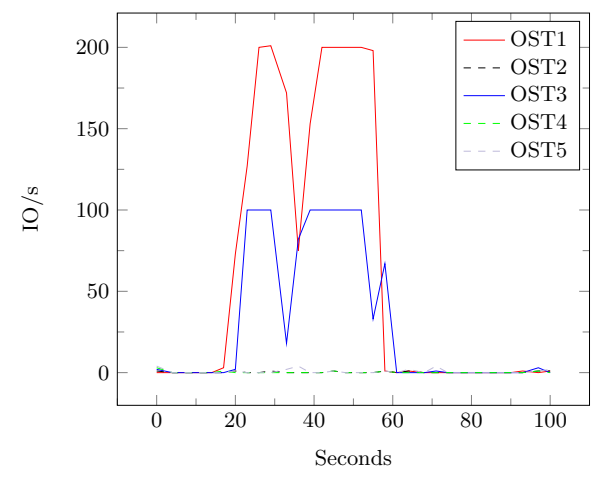

Figure 3: Loads on different OSTs

A typical problem in NUMA architectures is shown in Fig. 4. The figure depicts the number of NUMA hits that occur on the other node. This means that a process running on one socket has to use data allocated on the local memory of the other socket. We show two instances of the same problem in the figure. It can be seen how a non-optimal configuration leads to an execution time of over 60 seconds, while a better configuration takes just over 30 seconds to run. The application uses OpenMP to take advantage of the 16 cores available in the node (we used Stampede for these executions; each node in Stampede is dual socket eight-core Sandy-Bridge E5-2680 server node with 32 GB of memory) and numactl to control the NUMA policy.

One of the most common requirements from HPC users is to know how much memory their applications use on a given HPC resource. As previously described, REMORA is able to collect this information for either serial or parallel applications. Fig. 5 shows the total amount of memory used by 


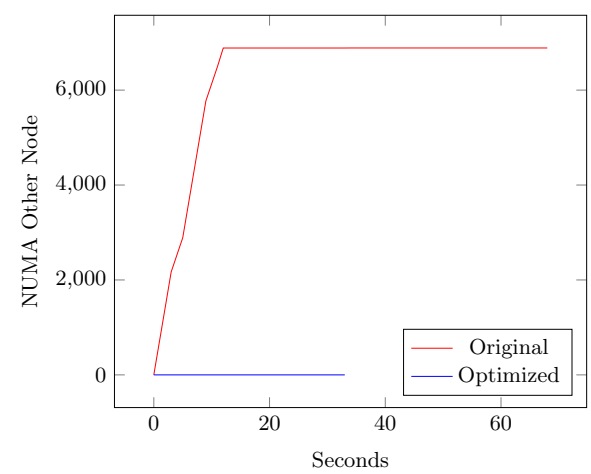

Figure 4: NUMA hits on the other node for two different executions

a serial application. This was an application that was executed on Stampede and that failed during the execution. The reason for that failure is depicted on the figure. Each node in Stampede has 32 GB of memory, but the operating system as well as other software need part of that memory. That results in approximately $28 \mathrm{~GB}$ of memory available for the user. The nodes are never shared between two users. In this case, the memory allocated increases during the execution of the application until it crashes because the out of memory killer is invoked. This particular application had a memory leak in the code that led the application to fail.

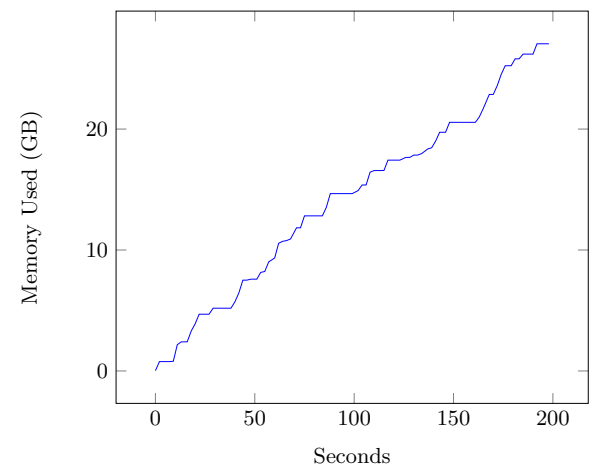

Figure 5: Memory utilization in one node

Fig. 6 shows the maximum amount of memory that has been allocated on each node by a parallel application. There is clearly a large imbalance between one node and the rest. This is a common scenario in a number of codes, either intentionally or by mistake. For example, this could represent a producer-consumer application where the producer method has larger memory requirements than the consumers. Or this could mean that the problem decomposition among the processes involved in the computation is not correct. The fact that three other processes have larger memory requirements than the remaining four processes seems to indicate the latter.

Some codes present imbalances not only in terms of memory, but also in terms of CPU utilization. Fig. 7 shows an OpenMP application that uses the static clause to split the computation among the cores. After an initialization phase,

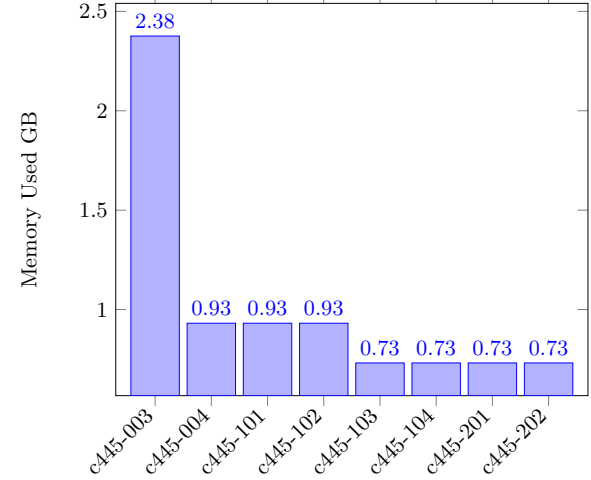

Figure 6: Memory utilization in different nodes

all the cores become active. It is possible to see in the figure how while some cores are fully utilized, the load of other cores remains at about $97 \%$. By switching the scheduling to dynamic the utilization of all the cores increases and remains at $100 \%$ during the execution of the code (see Fig. 8).

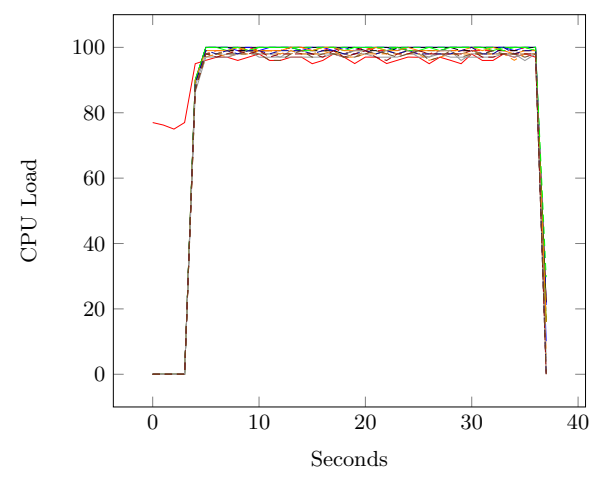

Figure 7: CPU imbalance for an application

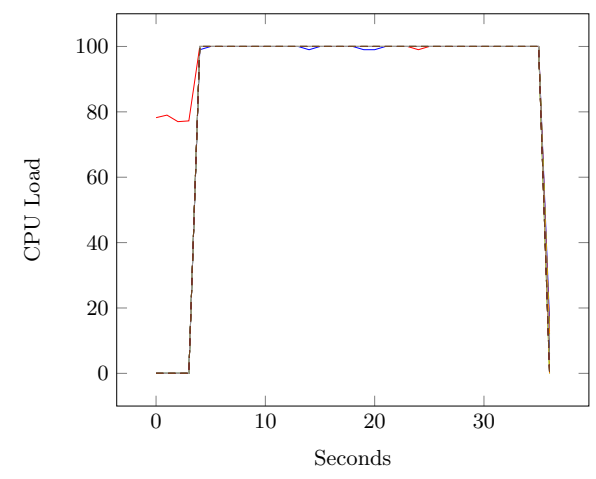

Figure 8: CPU balanced load for an application

\section{RELATED WORK}

To the best of our knowledge, there are no other tools that provide the same type of information and target the 
type of communities that REMORA does. There are, however, other approaches that allow users to collect some of the information that our tool provides. For example, the code peak-memusage [4] allows users to know the peak memory usage of an application once the application has finished. As described along this paper, this is very useful for users of HPC resources. However, the scope of that tool is smaller than what we propose in this paper.

The tool qmem [5] also offers very basic functionality that only considers the memory usage of a job. However, even though the use model is completely different to the one that we propose, it works in a similar manner to the way REMORA functions. In our case, we have built a tool that monitors the application that the user is running. In the case of qmem, the user has to call the tool from the login node with the identifier of the job of interest and qmem will collect the memory used in all the nodes that the job is using. Again, this is a tool with a much smaller scope than REMORA in terms of information provided to the user.

In [6], authors present a tool that aims at providing a similar type of information to the end user that what we present in this paper. Their approach depends on a middle layer for data collection and presents the results using a front-end interface. Because of the middle interface that they use, they can access a wider range of data but that also limits the portability and extensibility of the code when compared to REMORA.

The LDMS system developed at Sandia National Laboratories [7] is used by other tools [8], and provides much of the functionality that REMORA provides. However, it also requires a larger number of components to be installed and lacks the simplicity that the tool presented in this paper offers. We are also targeting end users that do not necessarily have an in-depth knowledge about computing.

Resource monitoring tools have typically been used by system administrators to monitor overall system utilization $[9,10]$. There are two weaknesses in the current implementation of these tools. First, in order to reduce system overhead their sampling frequency tends to be long, and may fail to capture important application behavior. Second, and perhaps most important, is the focus on system administration that those tools have. Few resource monitoring tools put the information in the hands of the end user [11], and most of them present an overwhelming amount of data in a manner that is not useful to a researcher trying to identify issues in his jobs. Some of the current tools include extremely scalable data collection [7] and excellent 2D and 3D data visualization [12], but still lack a mode that provides simplified advice to non-experts. Many other open source tools can be adapted to continuous system wide resource use profiling $[13,14]$ but none has a focus on data representation and actionable advice for the non-expert.

\section{CONCLUSION}

In this paper we have presented a tool that allows users and administrators of HPC resources to easily collect information about the computational resources used by a specific application. The data collected provides valuable insights about some of the most important requirements of the application and how it interacts with the system.

The tool is easy to use and puts special emphasis on collecting the most relevant information and presenting it in a clear format so that users can understand the implications of the data collected and how it can affect the execution of their applications. REMORA does not require code instrumentation or recompilation and is programming language agnostic.

All the tools that monitor the performance of an application or its interaction with the file system introduce some overhead. In many profilers this overhead can be very large. REMORA introduces a low overhead and it has been designed to minimize the computational resources that it requires.

As previously stated, there are some additional information that can be extracted with the data that the tool currently collects that will be added in the future. Correct striping of I/O operations is one of these insights, but CPU imbalance can be also easily analyzed and included in the tool.

We plan on supporting the MPI-T interface to be used in combination with some of the data that the tool already collects to provide users with better insights about the performance of parallel applications. This will imply transparently using MPI libraries that implement this interface without the intervention of the users.

The modular design allows users and administrator to extend the functionality of the tool. However, it is currently not possible to extend or modify the functionality of a system wide installation of REMORA without reinstalling it or without a local installation in the user account. We plan on allowing users to create new modules and to specify where those modules are located and the system wide installation of REMORA will be able to load and use those modules.

Finally, it is worth mentioning that currently REMORA stores all the data it collects in plain text files. This is very convenient for users, who can quickly and easily monitor the system usage. While we plan on keeping this format for the advantages just described, we expect to extend the functionality of the tool by also storing this data in a database. With the appropriate tools, it will make easier for users to compare the results of different executions and keep a repository with all the evaluations that have been performed in the past.

\section{Acknowledgment}

The authors gratefully acknowledge National Science Foundation support under award numbers ACI-1134872 (Stampede) and ACI 10-53575 (XSEDE).

\section{REFERENCES}

[1] C. Rosales, A. Gómez-Iglesias, and A. Predoehl, "REMORA: A resource monitoring tool for everyone," in Proceedings of the Second International Workshop on HPC User Support Tools, ser. HUST '15. New York, NY, USA: ACM, 2015, pp. 3:1-3:8. [Online]. Available: http://doi.acm.org/10.1145/2834996.2834999

[2] "REMORA repository," https://github.com/TACC/remora, accessed: 2016-04-25.

[3] "MP-LABS," https://github.com/carlosrosales/mplabs, accessed: 2016-04-25.

[4] "peak_memusage repository," https://github.com/davidedelvento/peak_memusage, 
accessed: 2016-04-25.

[5] "qmem repository,"

https://github.com/AnthonyDiGirolamo/qmem, accessed: 2016-04-25.

[6] C. Moore, P. Khalsa, T. Yilk, and M. Mason, "Monitoring high performance computing systems for the end user," in Cluster Computing (CLUSTER), 2015 IEEE International Conference on, Sept 2015, pp. $714-716$.

[7] A. Agelastos, B. A. Allan, J. M. Brandt, P. Cassella, J. Enos, J. Fullop, A. C. Gentile, S. Monk,

N. Naksinehaboon, J. Ogden, M. Rajan, M. T. Showerman, J. Stevenson, N. Taerat, and T. Tucker, "The lightweight distributed metric service: A scalable infrastructure for continuous monitoring of large scale computing systems and applications," in International Conference for High Performance Computing,

Networking, Storage and Analysis, SC 2014, New Orleans, LA, USA, November 16-21, 2014,

T. Damkroger and J. Dongarra, Eds. IEEE, 2014, pp. 154-165. [Online]. Available: http://dx.doi.org/10.1109/SC.2014.18

[8] A. Agelastos, B. Allan, J. Brandt, A. Gentile, S. Lefantzi, S. Monk, J. Ogden, M. Rajan, and J. Stevenson, "Toward rapid understanding of production hpc applications and systems," in Cluster Computing (CLUSTER), 2015 IEEE International Conference on, Sept 2015, pp. 464-473.

[9] M. Massie, B. Li, B. Nicholes, V. Vuksan, R. Alexander, J. Buchbinder, F. Costa, A. Dean, D. Josephsen, P. Phaal, and D. Pocock, Monitoring with Ganglia, 1st ed. O'Reilly Media, Inc., 2012.

[10] D. Josephsen, Building a monitoring infrastructure with Nagios. Prentice Hall PTR, 2007.

[11] E. Birngruber, P. Forai, and A. Zauner, "Total Recall: Holistic Metrics for Broad Systems Performance and User Experience Visibility in a Data-intensive Computing Environment," in Proceedings of the Second International Workshop on HPC User Support Tools, ser. HUST '15. New York, NY, USA: ACM, 2015, pp. 5:1-5:12. [Online]. Available: http://doi.acm.org/10.1145/2834996.2835001

[12] J. M. Brandt, B. J. Debusschere, A. C. Gentile, J. R. Mayo, P. P. Pébay, D. Thompson, and M. H. Wong, "OVIS-2: A robust distributed architecture for scalable RAS," in Parallel and Distributed Processing, 2008. IPDPS 2008. IEEE International Symposium on. IEEE, 2008, pp. 1-8.

[13] Online, "Performance Co-Pilot," https://http://www.pcp.io/, accessed: 2016-04-25.

[14] "HOPSA-Holistic Performance System Analysis," http://www.vi-hps.org/projects/hopsa/overview, accessed: 2016-04-25. 